Curr Opin Oncol. 2016 January ; 28(1): 18-25. doi:10.1097/CCO.0000000000000242.

\title{
Update on the Treatment of Hypothyroidism
}

\author{
Jacqueline Jonklaas \\ Division of Endocrinology, Georgetown University, Washington, DC
}

\begin{abstract}
Purpose of Review-Differentiated thyroid cancer is a malignancy that is rapidly increasing in frequency. As thyroidectomy plays a central role in the treatment of thyroid cancer, it is incumbent on physicians treating this patient group to be well versed in the intricacies of treating hypothyroidism.
\end{abstract}

Recent findings-Treatment of hypothyroidism may be refined by careful attention to dose selection, monitoring of therapy, and achievement of TSH goals that are specific to the individual patient's overall clinical situation. These goals are common not only to patients with a sole diagnosis of hypothyroidism, as discussed in the recent American Thyroid Association Guidelines, but also to patients with hypothyroidism in the setting of thyroid cancer. Several recent studies have illuminated our understanding of the benefits and risks of TSH suppression therapy in patients with differentiated thyroid cancer. Multiple studies of combination therapy with levothyroxine and liothyronine for treating hypothyroidism have not lead to a clear conclusion about its benefits over levothyroxine monotherapy. Animal studies have advanced our understanding of the altered serum and tissue milieu that characterizes levothyroxine monotherapy. Crossing the bridge from this translational research into clinical research using sustained release triiodothyronine preparations may ultimately enhance the health of our patients.

Summary-Continued refinement of our understanding of thyroid status and our ability to flawlessly implement thyroid hormone replacement is an active area of research.

\section{Keywords}

Hypothyroidism; levothyroxine replacement therapy; athyreotic; thyroid cancer; deiodinases; sustained release triiodothyronine

\section{Introduction}

Patients with a thyroid malignancy most commonly have undergone a total thyroidectomy, and are hence athyreotic, and dependent on thyroid hormone replacement. American Thyroid Association guidelines for the treatment of hypothyroidism were published in December 2014. The literature review for these guidelines was completed in December 2013. Although these guidelines focus on hypothyroidism without an accompanying thyroid

Address all correspondence and requests for reprints to: Jacqueline Jonklaas, Division of Endocrinology, Georgetown University, Suite 230, Bldg. D, 4000 Reservoir Road, NW, Washington, DC 20007., Phone number: 202687 2818, Fax number: 18774851479. jonklaaj@georgetown.edu.

Conflicts of interest: none. 
malignancy, many of the key points of these guidelines are pertinent to patients with thyroid cancer. The purpose of this review is to emphasize some of the key points from the hypothyroidism guidelines that are relevant to patients with thyroid cancer, and then discuss articles published since December 2013 that provide additional understanding or insight into these key points. Although many of the recent studies that are highlighted were conducted in individuals with autoimmune hypothyroidism, they have findings that are important for any individual taking thyroid hormone.

\section{The basics of treating hypothyroidism}

The basics of treating hypothyroidism include selection of the levothyroxine (LT4) dose, selecting a patient-appropriate serum thyrotropin (TSH) goal, and ensuring maintenance of that desired goal (1).

\section{Selection of LT4 dose}

Several approaches to estimating an appropriate LT4 dose haven been used in athyreotic individuals (1). These include weight-based or lean body mass-based formulae. Empiric dosing or formulae based on anthropometric characteristics have also been utilized.

a. Weight and Age-A weight-based LT4 dose in athyreotic patients is approximately 1.8 $\mathrm{mcg} / \mathrm{kg} /$ day for achieving a normal serum TSH, but $2.1-2.7 \mathrm{mcg} / \mathrm{kg} /$ day for achieving a low or suppressed TSH (1). There seems to be a tendency for older studies to find that higher doses are required which could be accounted for by multiple factors including differences in LT4 formulations. A recent study found that dose requirements for maintaining a slightly subnormal serum TSH of $0.21 \mathrm{mIU} / \mathrm{L}$ were $1.95 \mathrm{mcg} / \mathrm{kg} /$ day and $1.77 \mathrm{mcg} / \mathrm{kg} / \mathrm{day}$ in younger and older patients respectively (2). Empiric dosing based on ranges of weight, body mass index (BMI), and age have also been suggested, as well as formulae that include additional corrective factors combined with weight. A recent example of empiric dosing based on a combination of age and BMI for athyreotic patients in whom a normal TSH is desired is shown in table 1 (3). A recent example of a formula to achieve a normal TSH in athyreotic individuals is LT4 dose $(\mathrm{mcg} / \mathrm{kg} / \mathrm{day})=-0.018 \times \mathrm{BMI}+2.13$ (4). The latter authors found that incorporation of BMI into their formula better predicted the dose in overweight and underweight individuals. Prior studies have shown conflicting results about whether deiodinase polymorphisms affect LT4 dose requirement (1). A recent study of LT4 doses required for TSH suppression in patients with differentiated thyroid cancer (DTC) showed minimal affect of one of two polymorphisms in the uridine $5^{\prime}$-diphosphoglucuronyltransferase system responsible for metabolizing $\mathrm{T} 4$, and no effect of deiodinase polymorphisms (5). In a regression model, the authors found that age, sex, body weight, and TSH value accounted for 39\% of the variation in LT4 dose, whereas the identified haplotype only accounted for $2 \%$ of the variation (5).

b. Type of surgery-Although most patients with a cancer diagnosis undergo a total thyroidectomy, lobectomy is sometimes chosen for patients with low-risk papillary thyroid cancer. A recent study of patients undergoing lobectomy, $68 \%$ of whom were having surgery for a diagnosis of papillary thyroid, showed that a pre-operative TSH of $>2.5$ and 
the presence of thyroid peroxidase antibodies predicted the need to initiate LT4 to keep the serum TSH below 4-7 mIU/L (6).

\section{TSH goal}

The specific serum TSH that is desired in a patient is determined based on the balance of benefit and risks (1). Some degree of TSH suppression is beneficial for patients with higherrisk DTC, but this benefit must be balanced with the potential risks stemming from the individual patient's age and comorbidities.

a. Age-appropriate TSH Goals-TSH reference intervals are affected by several factors including patient age. The upper limit of the reference interval increases with age (1). Based on this it makes sense to modify the desired TSH goal for a patient with DTC depending on the patient's age, with a lesser degree of TSH suppression being the goal in an older patient. A recent study examining the effect of age, sex, and circadian rhythms on TSH reference intervals showed that the $97.5 \%$ confidence interval for the upper limit of the reference interval increased from 6.45 to $7.55 \mathrm{mIU} / \mathrm{L}$ with age (7). In addition, the increased TSH values in older individuals were thought to be due to a progressive increase in the nocturnal TSH surge (7).

b. Disease or condition-appropriate TSH Goals-The main diseases or conditions to be considered when deciding what TSH value is appropriate for a particular patient are whether the patient has DTC or not, and then additionally whether the patient has co-existent conditions that could potentially be worsened by maintaining a low TSH, such as cardiac arrhythmias or osteoporosis. If the patient has DTC, lower TSH values have traditionally been maintained for intermediate-risk and high-risk patients.

A recent publication from the National Thyroid Cancer Treatment Cooperative Study Group (NTCTCSG) addressed the issue of what TSH values improve the outcomes for patients with various DTC risk categories. This prospective study showed that maintaining serum TSH values in the normal-subnormal range improved the overall survival of patients with stage I-IV disease (see figures 1a-1d), increased the overall survival of patients with distant metastases (figure 1e), and also increased the disease free survival of patients with stage IIII disease (8). Aggressive TSH suppression to achieve a subnormal-undetectable TSH was not necessary to achieve these benefits. The beneficial effect of modest TSH suppression on survival appeared to be maintained over 3 years of follow up. In contrast with earlier reports from the NTCTCSG $(9,10)$, aggressive TSH suppression may not be warranted, even in patients diagnosed with distant metastatic disease during follow-up. These latter patients had decreased survival if their TSH values were maintained in the normal-elevated range, compared with the survival of those with undetectable-subnormal and subnormal-normal TSH values (8) However, there was no additional benefit of undetectable-subnormal TSH values compared with subnormal-normal values.

Several recent studies have examined the risks of TSH suppression. A recent study of patients with DTC with low to intermediate risk disease who were followed for 6 years found that those who were maintained with TSH values $<0.4 \mathrm{mIU} / \mathrm{L}$ had similar recurrence rates but more osteoporosis than those with TSH values $>0.4 \mathrm{mIU} / \mathrm{L}$ (11). Another recent 
study of elderly patients with DTC with 5 years of follow up who were retrospectively divided into 3 groups of TSH values of $<0.1,0.1-0.3$, or $0.3-0.5 \mathrm{mIU} / \mathrm{L}$ showed that recurrence rates were similar between the groups, but that complications increased in the groups with the lower TSH values. These complications included arrhythmias, osteoporosis, and anxiety/insomnia (12). Specifically examining bone health, a study in which the average TSH was $0.5 \mathrm{mIU} / \mathrm{L}$ in the one-year period after thyroidectomy, initiation of LT4 was associated with decreased bone mineral density in both the hip and the spine (13). However, this decline in bone density became insignificant after elapse of the initial one-year postoperative period. This finding is in keeping with the study by Wang et. al. (11), in which negative effects on bone health were no longer seen with maintenance of a median TSH of 1 mIU/L. In a cohort study of the Danish population, increased risk of hip fracture was seen during 7 years of follow up after a TSH value of $<0.3 \mathrm{mIU} / \mathrm{L}$ was documented, compared with those individuals who had a normal TSH value (14). This study excluded patients taking LT4, but would be applicable to patients with DTC if endogenous and exogenous hyperthyroidism had similar effects on the skeleton.

In contrast to these former studies showing an adverse effect of TSH suppression, one recent study of TSH suppression showed that an average serum TSH of $0.17 \mathrm{mIU} / \mathrm{L}$ over a 5 -year period was not associated with any cognitive impairment, as assessed using a battery of cognitive tests (15). However, the comparison group was euthyroid individuals not requiring LT4, rather than individuals who were euthyroid while taking LT4.

c. Out-of-goal TSH values-Iatrogenic thyroid disease occurs in a surprisingly high percentage of LT4-treated patients (1). In populations presumably being treated to achieve a normal TSH, approximately $20 \%$ of patients may be overtreated and another $20 \%$ undertreated, based on studies from 1993-2009 (16-19). A recent study from 2014 shows that this trend continues: 5 years after LT4 initiation, $20 \%$ of patients had TSH values over 4 $\mathrm{mIU} / \mathrm{L}$ and $10 \%$ had values $<0.5 \mathrm{mIU} / \mathrm{L}$ (20). Pregnancy is a time in which it generally agreed that specific TSH goals should be maintained. Of concern, a study of pregnant women found that $63 \%$ of women had TSH values $>2.5 \mathrm{mIU} / \mathrm{L}$ during the first trimester (21). Not only is this above the recommended target, but also the study investigators found that these elevated TSH values were associated with an increased risk of miscarriage.

The percentage of patients with DTC that are maintained with TSH values in specific ranges according to the patient's disease stage is often not reported. In a cohort of 3238 patients with DTC (8), where staging was classified using the NTCTCSG system, 30\% (427/1408) of patients with stage I disease had TSH values in the undetectable to subnormal range, whereas $10 \%$ (15/144) of patients with stage IV disease had TSH values in the normal to elevated range (see supplemental appendix 8 of cited article) (8). Moreover, in patients with distant metastases $6 \%$ (28/452) of patients had normal to elevated TSH values (see supplemental appendix 9 of cited article) (8). This recent publication found that moderate TSH suppression was beneficial across stages I-IV and in patients with distant metastases (see figures 1a-1e) (8). Thus, these data also suggest that LT4 therapy is often not optimized based on the patient's DTC risk category. 


\section{Maintenance of a specific TSH}

Once a specific TSH goal has been selected, there are several factors that can interfere with consistent maintenance of that particular target. These include altered absorption of LT4 caused by i) timing of LT4 ingestion, meals, beverages and medications, ii) altered transport of LT4 associated with medications, and iii) altered metabolism of LT4 caused by medications (1). Most of these situations result in the need for an increased LT4 dose to counteract their impact. However, recently some situations have been documented to either lower TSH or potentially reduce LT4 requirement.

a. Timing of LT4 administration-Several studies have examined the effect of the timing of LT4 on its absorption, as reflected in the resultant serum TSH (1). A recent review article (22) that compared the results of four studies which included bedtime dosing of LT4 in their study design (23-26) concluded that bedtime consumption of LT4 was a reasonable option for patients who wished to avoid taking their LT4 in close proximity to food, but also wanted a convenient regimen.

\section{b. Decreased LT4 absorption, altered transport and increased metabolism-}

Two recent "audits" have examined the medications associated with development of an elevated TSH and requirement for an increased dose of LT4. In one study, 21\% of patients requiring a LT4 dose of greater than $225 \mathrm{mcg}$ daily were found to also be taking medications commonly known to interfere with LT4 absorption or increase its metabolism, such as iron supplements, calcium supplements, anticonvulsants, and proton pump inhibitors (27). Altered timing of LT4 in those thought to have medication-induced impaired absorption reduced LT4 requirement by a mean of $31 \mathrm{mcg}$ daily. In the second study, patients initiated on a medication that was potentially a culprit medication were monitored for changes in serum TSH values. The medications noted to be associated with a significant increase in serum TSH were iron, calcium, proton pump inhibitors, and estrogen (28). A group of interfering medications that is particularly pertinent for patients with DTC is tyrosine kinase inhibitors. Many of these agents are associated with an increased requirement for LT4 in athyreotic individuals (see table 1 of cited article), possibly through several mechanisms including increased metabolism, possibly via induction of the type 3 deiodinase (29). In a recent study of children and adolescents with medullary thyroid cancer who were taking LT4, TSH values rose from the normal range to values ranging from $12.5-137 \mathrm{mIU} / \mathrm{L}$ with initiation of vandetanib treatment (30).

c. New levothyroxine formulations-Several case reports and non-randomized studies have suggested that liquid formulations of LT4 or formulations in which the LT4 is dissolved in glycerin and encased in a gelatin capsule may circumvent the impaired absorption of LT4 that may occur with tablet preparations. For patients receiving enteral feeding, liquid LT4 added directly to the feeding tube was associated with a similar serum TSH to that seen in another group of patients in whom the feeding was interrupted in order to administer crushed tablets (31). The former procedure was found to be more convenient by providers. In another study of patients taking proton pump inhibitors, switching to an oral solution was associated with a decrease in serum TSH from a mean of $5.4 \mathrm{mIU} / \mathrm{L}$ to 1.7 $\mathrm{mIU} / \mathrm{L}$, suggesting better absorption of the liquid preparation in patients being treated with 
proton pump inhibitors (32). A non-randomized study of patients with gastritis who had a stable serum TSH while taking LT4 tablets and who were switched to a lower dose of LT4 gel capsules, showed that two thirds of patients had a similar TSH on the lower dose, again suggesting better absorption of the gel capsule formulation (33). Another non-randomized study suggests that the serum TSH achieved by LT4 gel capsules is not affected by the timing with respect to breakfast (34). If the findings of these studies are bolstered by randomized controlled studies in the future, these LT4 formulations may prove very convenient for hypothyroid patients.

d. Medications associated with lowered TSH values-At least 3 medications have been recently associated with lowered serum TSH values in patients taking LT4. These are statins, metformin, and vitamin C. In an observational study patient started on statins were noted to have a very small decrease in their TSH values from 1.62 to $1.45 \mathrm{mIU} / \mathrm{L}$ ( $\mathrm{p}$ value 0.01 ) after beginning a statin (28). A meta-analysis of 4 studies examining the effect of metformin on serum TSH found a small but statistically significant decline in TSH after metformin therapy was started (35). Another study from 2014 also confirmed this TSHlowering property of metformin (36). However, a study of patients with DTC whose LT4 dose was lowered by $33 \%$ coincidentally with metformin or placebo initiation, showed that both groups of patients experienced a similar increase in serum TSH from a subnormal value to $3.1 \mathrm{mIU} / \mathrm{L}$ (37), illustrating the very small, clinically insignificant, magnitude of the metformin effect. The mechanism of TSH-lowering effect of statins and metformin has not been fully elucidated, but seems unlikely to be due to enhanced absorption $(28,36)$. Vitamin $\mathrm{C}$, on the other hand, may improve LT4 absorption in patients with gastritis (38). In the latter study patients with elevated TSH levels while taking LT4 had lowering of their TSH values from median of 11.1 to $4.2 \mathrm{mIU} / \mathrm{L}$ when they continued the same dose of LT4 with the addition of vitamin $\mathrm{C}$ solution (38).

\section{Future directions in the treatment of hypothyroidism}

One of the more complex issues to be considered when treating hypothyroidism is how to ensure that patients undergoing treatment are restored to optimal health (1). Inherent in considering this issue are three related questions. The first is whether quality of life is decreased in patients with hypothyroidism who are taking LT4 monotherapy, the second is whether combination therapy with LT4 and liothyronine (LT3) is able to reverse the quality of life impairments, and the third is whether the inherent problem with LT4 monotherapy is the failure of deiodinases to adequately compensate for the high T4/T3 ratio in athyreotic patients.

\section{Quality of Life}

Several studies have shown decreased psychological well-being, more thyroid-related symptoms, more dissatisfaction, increased fatigue, more cognitive impairment, and decreased well-being in patients being treated for hypothyroidism, compared with various control groups (1) A recent review discussed the issue of fatigue in patients with DTC and concluded that most evidence was epidemiologic in nature, with diverse control populations, unclear relationship with biochemical indices, and lack of randomized, controlled trials of 
treatment (39). For a discussion of quality of life in patients with DTC also see the review by Dr. Sawka in this issue. A recent study has also shown that patients with hypothyroidism are at more risk of being diagnosed with a psychiatric disorder and being treated with antidepressive or anti-anxiety medications (40).

\section{Combination therapy}

The studies reported thus far have not clearly shown benefits of combination therapy on health-related quality of life, mood, or neurocognitive functioning (1). When combining the patients participating in cross-over studies, similar numbers of patients had preference for combination therapy $(n=128)$ as had no preference $(n=101)$. Combining the numbers from the parallel design studies, more patients had no preference $(n=573)$ than had a preference for combination therapy $(\mathrm{n}=130)(1)$. Although a metaanalysis did not find any association between positive outcomes with the use of combination therapy and the athyreotic state (41), there were relatively few athyreotic patients within the populations being studied. Drawing conclusions from current studies is confounded by their heterogeneity. This heterogeneity involves study design, outcome measures, and results, and includes use of different $\mathrm{T} 3$ doses and frequency of dosing. When a clinical trial of a sustained release T3 preparation, given in a dose that is physiologic for humans, has been conducted, this will greatly advance the field. A metabolite of T3, T3 sulfate, has been shown to provide steady T3 levels for at least 48 hours, but this property has only been demonstrated in profoundly hypothyroid patients undergoing a thyroid hormone withdrawal protocol for treatment of DTC (42).

\section{The role of deiodinases in ensuring euthyroidism}

A much-cited retrospective study raised the possibility that a positive response to use of combination therapy may be associated with the presence of a type 2 deiodinase polymorphism (43). Although prospective clinical trials that examine the outcomes of combination therapy based on deiodinase polymorphism status or activity of deiodinases have not yet been published, several pre-clinical investigations have focused on the issue of deiodinase activity. Mathematical modeling suggests decreased deiodinase activity, as well as decreased free T3/free T4 ratios, in LT4-treated patients, with decreases being more noteable in athyreotic patients (44). As a complement to prior animal studies by EscobarMorreale et al $(45,46)$, a recent study conducted in rats suggested impairment of type 2 deiodinase activity in the whole body during LT4 monotherapy due to deiodinase inactivation, compared with maintenance of deiodinase activity in the hypothalamus. The lesser degree of activation in the hypothalamus leads to efficient $\mathrm{T} 3$ production in the hypothalamus and normalization of TSH before T3 normalizes in the rest of the body. Accompanying the inactivation of type 2 deiodinase in other tissues, lower serum T3 and higher T4/T3 ratios were seen in rats during monotherapy compared with combination therapy employing a subcutaneous slow release T3 pellet (47).

\section{Conclusion}

Despite the advances made in the treatment of hypothyroidism, there are many knowledge gaps that must be surmounted in order to ensure that quality of life is optimized in all our patients with hypothyroidism. This is particularly necessary in patients that also have DTC, 
in whom quality of life may also be compromised due to knowledge of having a malignancy and the additional therapy, monitoring and expenses that this requires. LT4 monotherapy has been refined in many ways. We understand the factors affecting dose requirement, the means by which to maintain a therapeutic serum TSH, and the importance of considering the desired TSH goal on an individual patient basis. We know that combination therapy with our current liothyronine preparations, when given as a once or twice daily dose, does not clearly improve patient outcomes. We eagerly await a sustained release liothyronine preparation that can be tested in athyreotic patients with the hope that the milieu of all tissues can be rendered euthyroid and that patient satisfaction with therapy and patient health outcomes can be concomitantly improved.

\section{Acknowledgments}

Financial support and sponsorship. JJ is supported by National Institutes of Health grants R01AG033867 and UL1TR000101.

\section{Abbreviations}

$\begin{array}{ll}\text { TSH, LT4 } & \begin{array}{l}\text { levothyroxine, thyrotropin } \\ \text { BMI }\end{array} \\ \text { DTC } & \text { differentiated thyroid cancer } \\ \text { NTCTCSG } & \text { National Thyroid Cancer Treatment Cooperative Study Group } \\ \text { T4 } & \text { thyroxine } \\ \text { T3 } & \text { triiodothyronine } \\ \text { FT4 } & \text { free thyroxine } \\ \text { FT3 } & \text { free triiodothyronine }\end{array}$

\section{References}

*1. Jonklaas J, Bianco AC, Bauer AJ, Burman KD, Cappola AR, Celi FS, et al. Guidelines for the treatment of hypothyroidism: prepared by the american thyroid association task force on thyroid hormone replacement. Thyroid : official journal of the American Thyroid Association. 2014; 24(12):1670-751. These guidelines focus on the treatment of hypothyroidism, with special attention to a discussion of combination therapy with T4 and T3. [PubMed: 25266247]

2. Del Duca SC, Santaguida MG, Brusca N, Gatto I, Cellini M, Gargano L, et al. Individually-Tailored Thyroxine Requirement in the Same Patients before and after Thyroidectomy: A Longitudinal Study. European journal of endocrinology/European Federation of Endocrine Societies. 2015

*3. Di Donna V, Santoro MG, de Waure C, Ricciato MP, Paragliola RM, Pontecorvi A, et al. A new strategy to estimate levothyroxine requirement after total thyroidectomy for benign thyroid disease. Thyroid : official journal of the American Thyroid Association. 2014; 24(12):1759-64. This study shows that both BMI and age affect LT4 dosage requirement in athyreotic patients. [PubMed: 25268754]

*4. Ojomo KA, Schneider DF, Reiher AE, Lai N, Schaefer S, Chen H, et al. Using body mass index to predict optimal thyroid dosing after thyroidectomy. Journal of the American College of Surgeons. 2013; 216(3):454-60. This study shows improved prediction of LT4 dose in athyreotic patients using a formula that incorporates BMI. [PubMed: 23318118] 
*5. Santoro AB, Vargens DD, de Barros Filho MC, Bulzico DA, Kowalski LP, Meirelles RM, et al. Effect of UGT1A1, UGT1A3, DIO1 and DIO2 polymorphisms on L-thyroxine doses required for TSH suppression in patients with differentiated thyroid cancer. British journal of clinical pharmacology. 2014; 78(5):1067-75. This study investigates whether polymorphisms in the genes encoding enzymes involved in deiodinating and metabolizing T4 affect LT4 dose requirement, and find minimal impact of one of the polymorphisms. [PubMed: 24910925]

6. Lee DY, Seok J, Jeong WJ, Ahn SH. Prediction of thyroid hormone supplementation after thyroid lobectomy. The Journal of surgical research. 2015; 193(1):273-8. [PubMed: 25088372]

*7. Ehrenkranz J, Bach PR, Snow GL, Schneider A, Lee JL, Ilstrup S, et al. Circadian and Circannual Rhythms in Thyroid Hormones: Determining the TSH and Free T4 Reference Intervals Based Upon Time of Day, Age, and Sex. Thyroid : official journal of the American Thyroid Association. 2015 This study shows that the progressive increase in the upper limit of the TSH reference interval with age is due to an increase in the amplitude of the nocturnal TSH surge.

**8. Carhill AA, Litofsky DR, Ross DS, Jonklaas J, Cooper DS, Brierley JD, et al. Long-Term Outcomes Following Therapy in Differentiated Thyroid Carcinoma: NTCTCS Registry Analysis 1987-2012. The Journal of clinical endocrinology and metabolism. 2015:JC20151346. This prospective study shows that moderate TSH suppression improves the outcomes of patients with all stages of thyroid cancer, including those with distant metastases, but that aggressive TSH suppresion offers no advantage.

9. Cooper DS, Specker B, Ho M, Sperling M, Ladenson PW, Ross DS, et al. Thyrotropin suppression and disease progression in patients with differentiated thyroid cancer: results from the National Thyroid Cancer Treatment Cooperative Registry. Thyroid : official journal of the American Thyroid Association. 1998; 8(9):737-44. [PubMed: 9777742]

10. Jonklaas J, Sarlis NJ, Litofsky D, Ain KB, Bigos ST, Brierley JD, et al. Outcomes of patients with differentiated thyroid carcinoma following initial therapy. Thyroid : official journal of the American Thyroid Association. 2006; 16(12):1229-42. [PubMed: 17199433]

*11. Wang LY, Smith AW, Palmer FL, Tuttle RM, Mahrous A, Nixon IJ, et al. Thyrotropin suppression increases the risk of osteoporosis without decreasing recurrence in ATA low- and intermediate-risk patients with differentiated thyroid carcinoma. Thyroid : official journal of the American Thyroid Association. 2015; 25(3):300-7. In this study TSH suppression in patients with low-risk and intermediate-risk thyroid cancer did not decrease their risk of disease recurrence, but did increase their risk of osteoporosis. [PubMed: 25386760]

12. Xia Q, Dong S, Bian PD, Wang J, Li CJ. Effects of endocrine therapy on the prognosis of elderly patients after surgery for papillary thyroid carcinoma. European archives of oto-rhinolaryngology : official journal of the European Federation of Oto-Rhino-Laryngological Societies. 2015

13. Kim MK, Yun KJ, Kim MH, Lim DJ, Kwon HS, Song KH, et al. The effects of thyrotropinsuppressing therapy on bone metabolism in patients with well-differentiated thyroid carcinoma. Bone. 2015; 71:101-5. [PubMed: 25445448]

14. Abrahamsen B, Jorgensen HL, Laulund AS, Nybo M, Brix TH, Hegedus L. Low serum thyrotropin level and duration of suppression as a predictor of major osteoporotic fractures-the OPENTHYRO register cohort. Journal of bone and mineral research : the official journal of the American Society for Bone and Mineral Research. 2014; 29(9):2040-50.

15. Moon JH, Ahn S, Seo J, Han JW, Kim KM, Choi SH, et al. The effect of long-term thyroidstimulating hormone suppressive therapy on the cognitive function of elderly patients with differentiated thyroid carcinoma. The Journal of clinical endocrinology and metabolism. 2014; 99(10):3782-9. [PubMed: 25029415]

16. Parle JV, Franklyn JA, Cross KW, Jones SR, Sheppard MC. Thyroxine prescription in the community: serum thyroid stimulating hormone level assays as an indicator of undertreatment or overtreatment. The British journal of general practice : the journal of the Royal College of General Practitioners. 1993; 43(368):107-9. [PubMed: 8323787]

17. Somwaru LL, Arnold AM, Joshi N, Fried LP, Cappola AR. High frequency of and factors associated with thyroid hormone over-replacement and under-replacement in men and women aged 65 and over. The Journal of clinical endocrinology and metabolism. 2009; 94(4):1342-5. [PubMed: 19126628] 
18. Canaris GJ, Manowitz NR, Mayor G, Ridgway EC. The Colorado thyroid disease prevalence study. Archives of internal medicine. 2000; 160(4):526-34. [PubMed: 10695693]

19. Hollowell JG, Staehling NW, Flanders WD, Hannon WH, Gunter EW, Spencer CA, et al. Serum TSH, T(4), and thyroid antibodies in the United States population (1988 to 1994): National Health and Nutrition Examination Survey (NHANES III). The Journal of clinical endocrinology and metabolism. 2002; 87(2):489-99. [PubMed: 11836274]

20. Taylor PN, Iqbal A, Minassian C, Sayers A, Draman MS, Greenwood R, et al. Falling threshold for treatment of borderline elevated thyrotropin levels-balancing benefits and risks: evidence from a large community-based study. JAMA internal medicine. 2014; 174(1):32-9. [PubMed: 24100714]

21. Taylor PN, Minassian C, Rehman A, Iqbal A, Draman MS, Hamilton W, et al. TSH levels and risk of miscarriage in women on long-term levothyroxine: a community-based study. The Journal of clinical endocrinology and metabolism. 2014; 99(10):3895-902. [PubMed: 25057882]

22. Geer M, Potter DM, Ulrich H. Alternative schedules of levothyroxine administration. American journal of health-system pharmacy : AJHP : official journal of the American Society of HealthSystem Pharmacists. 2015; 72(5):373-7. [PubMed: 25694412]

23. Bach-Huynh TG, Nayak B, Loh J, Soldin S, Jonklaas J. Timing of levothyroxine administration affects serum thyrotropin concentration. The Journal of clinical endocrinology and metabolism. 2009; 94(10):3905-12. [PubMed: 19584184]

24. Bolk N, Visser TJ, Kalsbeek A, van Domburg RT, Berghout A. Effects of evening vs morning thyroxine ingestion on serum thyroid hormone profiles in hypothyroid patients. Clinical endocrinology. 2007; 66(1):43-8. [PubMed: 17201800]

25. Bolk N, Visser TJ, Nijman J, Jongste IJ, Tijssen JG, Berghout A. Effects of evening vs morning levothyroxine intake: a randomized double-blind crossover trial. Archives of internal medicine. 2010; 170(22):1996-2003. [PubMed: 21149757]

26. Rajput R, Chatterjee S, Rajput M. Can Levothyroxine Be Taken as Evening Dose? Comparative Evaluation of Morning versus Evening Dose of Levothyroxine in Treatment of Hypothyroidism. Journal of thyroid research. 2011; 2011:505239. [PubMed: 21785690]

27. Robertson HM, Narayanaswamy AK, Pereira O, Copland SA, Herriot R, McKinlay AW, et al. Factors contributing to high levothyroxine doses in primary hypothyroidism: an interventional audit of a large community database. Thyroid : official journal of the American Thyroid Association. 2014; 24(12):1765-71. [PubMed: 25203248]

28. Irving SA, Vadiveloo T, Leese GP. Drugs that interact with levothyroxine: an observational study from the Thyroid Epidemiology, Audit and Research Study (TEARS). Clinical endocrinology. 2015; 82(1):136-41. [PubMed: 25040647]

29. Illouz F, Braun D, Briet C, Schweizer U, Rodien P. Endocrine side-effects of anti-cancer drugs: thyroid effects of tyrosine kinase inhibitors. European journal of endocrinology/European Federation of Endocrine Societies. 2014; 171(3):R91-9. [PubMed: 24833135]

*30. Lodish M, Gkourogianni A, Bornstein E, Sinaii N, Fox E, Chuk M, et al. Patterns of thyroid hormone levels in pediatric medullary thyroid carcinoma patients on vandetanib therapy. International journal of pediatric endocrinology. 2015; 2015(1):3. This study shows that athyreotic pediatric patient require an increase in LT4 to maintain euthyroidism following initiation of vantetanib. [PubMed: 25972901]

31. Pirola I, Daffini L, Gandossi E, Lombardi D, Formenti A, Castellano M, et al. Comparison between liquid and tablet levothyroxine formulations in patients treated through enteral feeding tube. Journal of endocrinological investigation. 2014; 37(6):583-7. [PubMed: 24789541]

32. Vita R, Saraceno G, Trimarchi F, Benvenga S. Switching levothyroxine from the tablet to the oral solution formulation corrects the impaired absorption of levothyroxine induced by proton-pump inhibitors. The Journal of clinical endocrinology and metabolism. 2014; 99(12):4481-6. [PubMed: 25259910]

33. Santaguida MG, Virili C, Del Duca SC, Cellini M, Gatto I, Brusca N, et al. Thyroxine softgel capsule in patients with gastric-related T4 malabsorption. Endocrine. 2015; 49(1):51-7. [PubMed: 25595886] 
34. Cappelli C, Pirola I, Gandossi E, Formenti A, Castellano M. Oral liquid levothyroxine treatment at breakfast: a mistake? European journal of endocrinology/European Federation of Endocrine Societies. 2014; 170(1):95-9. [PubMed: 24123095]

35. Lupoli R, Di Minno A, Tortora A, Ambrosino P, Lupoli GA, Di Minno MN. Effects of treatment with metformin on TSH levels: a meta-analysis of literature studies. The Journal of clinical endocrinology and metabolism. 2014; 99(1):E143-8. [PubMed: 24203069]

36. Fournier JP, Yin H, Yu OH, Azoulay L. Metformin and low levels of thyroid-stimulating hormone in patients with type 2 diabetes mellitus. CMAJ : Canadian Medical Association journal = journal de l'Association medicale canadienne. 2014; 186(15):1138-45.

37. Mousavi Z, Dourandish L, Rokni H, Sadeghi R, Rasoul Zakavi S. Effects of short-term metformin therapy associated with levothyroxine dose decrement on TSH and thyroid hormone levels in patients with thyroid cancer. Minerva endocrinologica. 2014; 39(1):59-65. [PubMed: 24513605]

*38. Jubiz W, Ramirez M. Effect of vitamin C on the absorption of levothyroxine in patients with hypothyroidism and gastritis. The Journal of clinical endocrinology and metabolism. 2014; 99(6):E1031-4. This study found that in patients with gastritis and an elevated serum TSH while taking LT4 therapy, consumption of vitamin C lowered the TSH. [PubMed: 24601693]

39. Sawka AM, Naeem A, Jones J, Lowe J, Segal P, Goguen J, et al. Persistent posttreatment fatigue in thyroid cancer survivors: a scoping review. Endocrinology and metabolism clinics of North America. 2014; 43(2):475-94. [PubMed: 24891173]

40. Thvilum M, Brandt F, Almind D, Christensen K, Brix TH, Hegedus L. Increased psychiatric morbidity before and after the diagnosis of hypothyroidism: a nationwide register study. Thyroid : official journal of the American Thyroid Association. 2014; 24(5):802-8. [PubMed: 24383722]

41. Grozinsky-Glasberg S, Fraser A, Nahshoni E, Weizman A, Leibovici L. Thyroxinetriiodothyronine combination therapy versus thyroxine monotherapy for clinical hypothyroidism: meta-analysis of randomized controlled trials. The Journal of clinical endocrinology and metabolism. 2006; 91(7):2592-9. [PubMed: 16670166]

42. Santini F, Giannetti M, Ricco I, Querci G, Saponati G, Bokor D, et al. Steady-State Serum T3 Concentrations for 48 Hours Following the Oral Administration of a Single Dose of 3,5,3'Triiodothyronine Sulfate (T3S). Endocrine practice : official journal of the American College of Endocrinology and the American Association of Clinical Endocrinologists. 2014; 20(7):680-9.

43. Panicker V, Saravanan P, Vaidya B, Evans J, Hattersley AT, Frayling TM, et al. Common variation in the DIO2 gene predicts baseline psychological well-being and response to combination thyroxine plus triiodothyronine therapy in hypothyroid patients. The Journal of clinical endocrinology and metabolism. 2009; 94(5):1623-9. [PubMed: 19190113]

44. Hoermann R, Midgley JE, Larisch R, Dietrich JW. Integration of Peripheral and Glandular Regulation of Triiodothyronine Production by Thyrotropin in Untreated and Thyroxine-Treated Subjects. Hormone and metabolic research $=$ Hormon- und Stoffwechselforschung $=$ Hormones et metabolisme. 2015

45. Escobar-Morreale HF, Obregon MJ, Escobar del Rey F, Morreale de Escobar G. Replacement therapy for hypothyroidism with thyroxine alone does not ensure euthyroidism in all tissues, as studied in thyroidectomized rats. The Journal of clinical investigation. 1995; 96(6):2828-38. [PubMed: 8675653]

46. Escobar-Morreale HF, del Rey FE, Obregon MJ, de Escobar GM. Only the combined treatment with thyroxine and triiodothyronine ensures euthyroidism in all tissues of the thyroidectomized rat. Endocrinology. 1996; 137(6):2490-502. [PubMed: 8641203]

**47. Werneck de Castro JP, Fonseca TL, Ueta CB, McAninch EA, Abdalla S, Wittmann G, et al. Differences in hypothalamic type 2 deiodinase ubiquitination explain localized sensitivity to thyroxine. The Journal of clinical investigation. 2015; 125(2):769-81. This study in rats shows that inactivation of the type 2 deiodinase occurs to different extents in different tissues, with the lesser degree of activation in the hypothalamus accounting for efficient T3 production in the hypothalamus and normalization of TSH before T3 normalizes in the rest of the body. [PubMed: 25555216] 


\section{Key points}

Hypothyroidism is a life-long condition in athyreotic patients, so optimization of therapy is critical.

The complex interaction of factors such as patient age, sex, and weight in determining levothyroxine dose requirement is being increasing appreciated.

New liquid levothyroxine preparations presently being studied may ease the burden for patients attempting to adhere to a levothyroxine regimen that maintains a steady serum TSH value.

The benefits and risk of TSH suppression therapy should be carefully considered on an individual patient basis.

There is hope, based on animal studies, that a sustained release triiodothyronine preparation may reverse the disappointing trend for combination studies to fail to show a clear improvement in patient outcomes. 


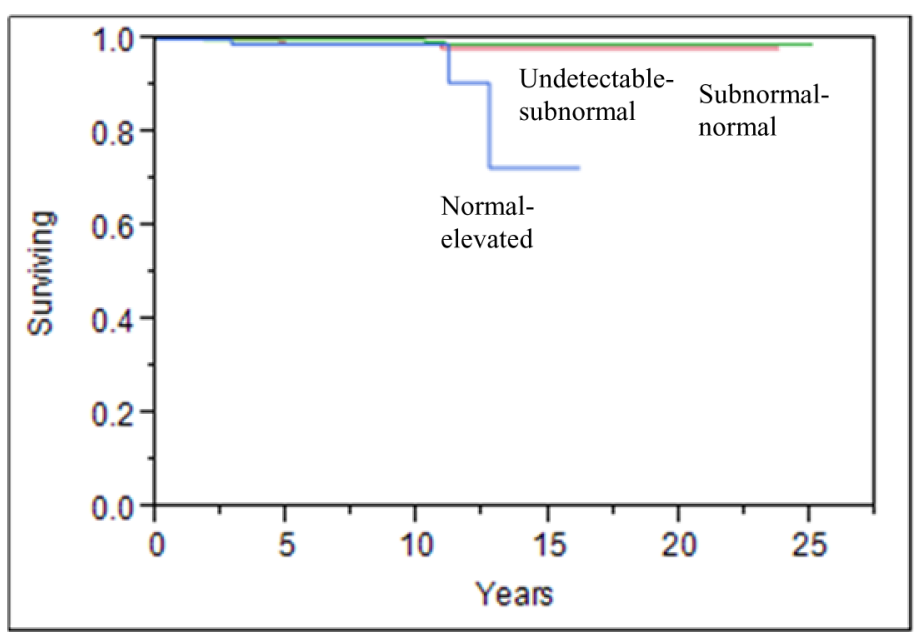

Fig.1a.

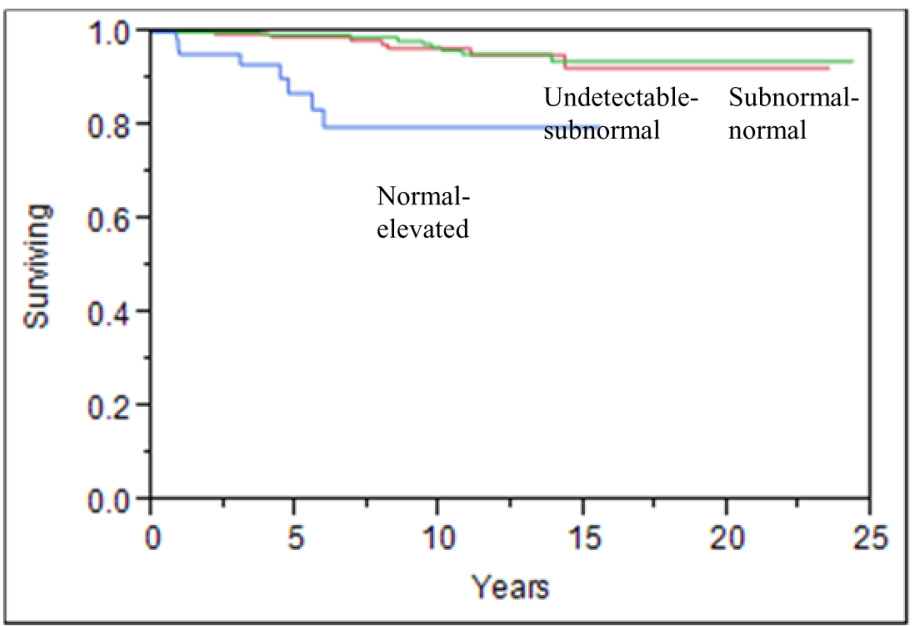

Fig.1b. 


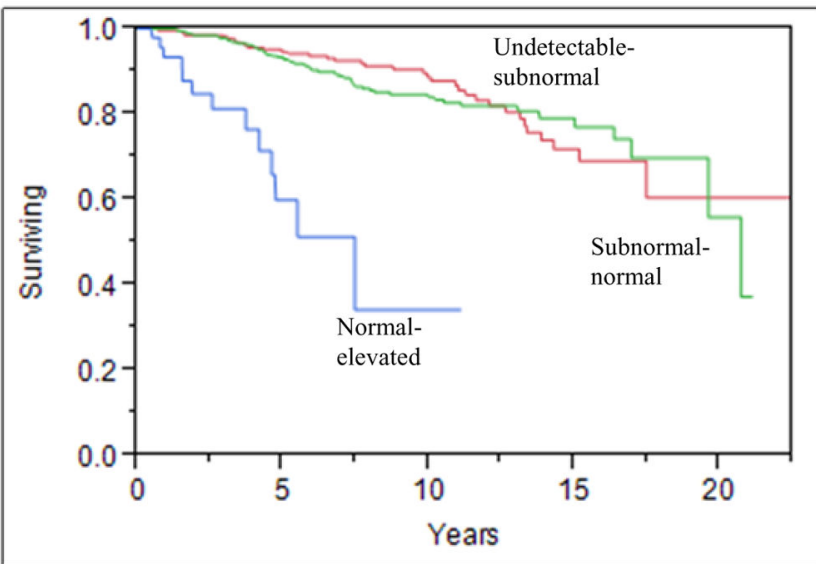

Fig.1c.

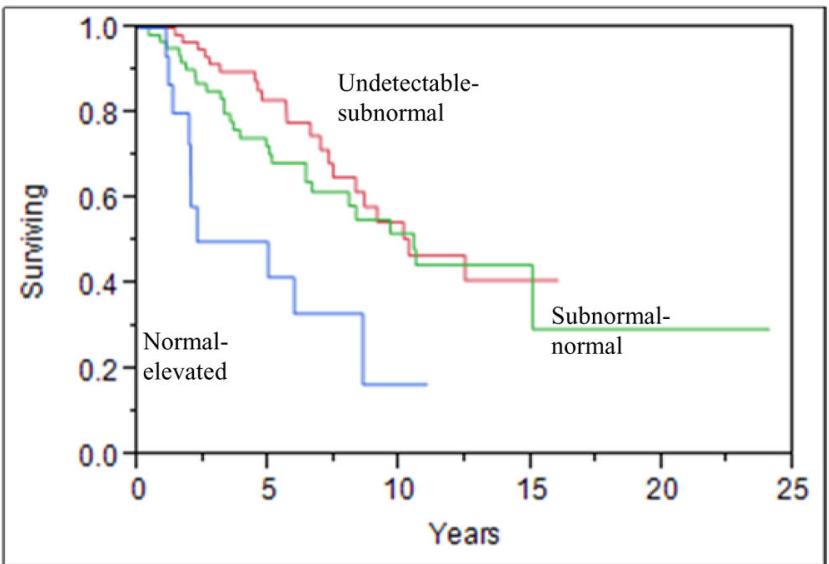

Fig.1d. 


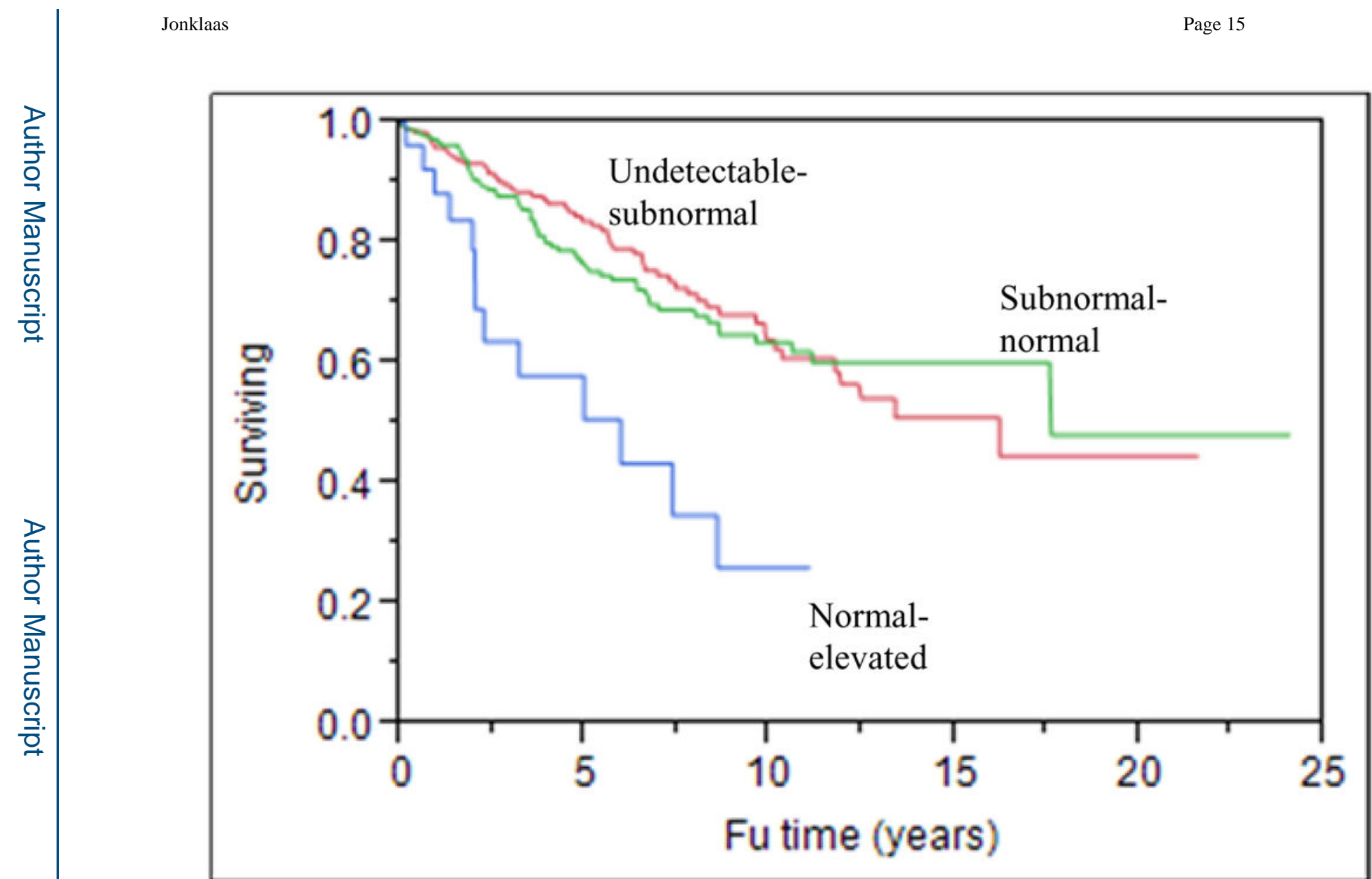

Fig. 1e.

Fig. 1.

Fig. 1a. Product-limit estimates of overall survival according to mean TSH category STAGE I patients $(\mathrm{p}=0.002)$

Fig.1b. Product-limit estimates of overall survival according to mean TSH category STAGE II patients $(\mathrm{p}<0.0001)$

Fig.1c. Product-limit estimates of overall survival according to mean TSH category STAGE III patients $(\mathrm{p}<0.0001)$

Fig.1d. Product-limit estimates of overall survival according to mean TSH category STAGE IV patients $(\mathrm{p}=0.003)$

Fig. 1e. Product-limit estimates of overall survival according to mean TSH score category after diagnosis of distant metastases $(\mathrm{p}=0.0003)$

With permission from (8) 


\section{Table 1}

LT4 dosing in athyreotic individuals

\begin{tabular}{|l|l|l|l|l|}
\hline Reference & \multicolumn{4}{|l|}{ Estimates of LT4 dose requirement } \\
\hline \multirow{4}{*}{$(3)$} & \multicolumn{4}{|l|}{ Empiric dosing based on age and BMI ranges } \\
\cline { 2 - 5 } & LT4 dose (mcg/kg/day) & BMI $\_3$ & BMI 24-28 & BMI >28 \\
\cline { 2 - 5 } & Age $\leq 40$ yrs & 1.8 & 1.7 & 1.6 \\
\cline { 2 - 5 } & Age 41-55 years & 1.7 & 1.6 & 1.5 \\
\cline { 2 - 5 } & Age $>55$ yrs & 1.6 & 1.5 & 1.4 \\
\hline \multirow{4}{*}{$(4)$} & Formula-based dosing \\
\hline & LT4 (mcg/kg/day) $=-0.018 \times$ BMI + 2.13 \\
\hline
\end{tabular}

With permission from (3) 